\title{
Le débat universitaire en France
}

De la montée des tensions à la reconfiguration du paysage universitaire

The university debate in France. From the rise of tensions to the remodelling of the academic scene

El debate universitario en Francia. Desde la subida de las tensiones hasta la remodelación del paisaje universitario

\section{Patricia Pol}

\section{OpenEdition \\ Journals}

Édition électronique

URL : http://journals.openedition.org/ries/255

DOI : $10.4000 /$ ries. 255

ISSN : 2261-4265

Éditeur

Centre international d'études pédagogiques

Édition imprimée

Date de publication : 1 septembre 2007

Pagination : 87-97

ISBN : 978-2-85420-570-1

ISSN : $1254-4590$

\section{Référence électronique}

Patricia Pol, «Le débat universitaire en France », Revue internationale d'éducation de Sèvres [En ligne] 45 | septembre 2007, mis en ligne le 23 juin 2011, consulté le 19 avril 2019. URL : http:// journals.openedition.org/ries/255; DOI : 10.4000/ries.255 


\section{Le débat universitaire en France}

\section{De la montée des tensions à la reconfiguration du paysage universitaire}

\section{Patricia Pol}

Présenter le système d'enseignement supérieur français est une gageure, selon nombre de ses acteurs. Éclaté entre des types d'établissements très différents relevant d'autorités nationales multiples, hiérarchisé entre des universités et des grandes écoles, des filières dites générales et d'autres professionnelles, des organismes de recherche puissants, des équipes mixtes de recherche, le système serait plus complexe, moins lisible et moins efficace qu'ailleurs. Des ouvrages récents parus sur l'université française et publiés par des universitaires parlent de "misère ", de "destruction organisée du savoir », de "grande illusion» (Jourde, 2007). L'université serait non seulement à la dérive, mais incapable de s'organiser pour résister aux objectifs à court terme de nos responsables politiques. Si cette vision reflète une réalité parfois incontestable, force est de constater que depuis plus de trente ans, l'université française a connu des évolutions considérables, témoignant ainsi de ses réelles capacités de changement tout en maintenant ses croyances fortes en des valeurs dites académiques.

Fortement ancrée dans des traditions historiques qui pèsent encore lourdement sur l'ensemble de notre système, la France traverse les débats universitaires contemporains avec les mêmes difficultés que les autres pays européens. Les discours sur la nécessaire adaptation aux pressions de l'environnement devenu plus global et concurrentiel ont conduit et vont encore conduire à des ajustements stratégiques et à des changements de pratiques, tant au plan de la politique universitaire nationale qu'au niveau des institutions elles-mêmes. S'adapter à la nouvelle économie du savoir amènerait à repenser l'université et ses missions, selon les multiples rapports produits par l'OCDE depuis les années quatre-vingts (Milot, 2001). Le nouveau management public (NPM) serait la solution pour moderniser les modes de gouvernance des universités comme les exemples britannique, néerlandais, autrichien ou allemand l'illustreraient (Schimank, 2007).

Parallèlement, l'émergence de dynamiques plus collectives a été impulsée dans un contexte français. Ne doit-on pas en effet le Processus de Bologne à l'initiative du ministre Claude Allègre en mai 1998, qui, prenant le symbole de l'anniversaire de la Sorbonne, posa les bases de la création d'un espace européen de l'enseignement supérieur? Qui aurait alors pu croire que ce processus allait impliquer moins de dix ans plus tard quarante-six ministres, des milliers 
d'établissements et plus de trente millions d'étudiants bien au-delà des frontières de l'Union européenne? Mais en même temps, en 2007, les universitaires français, épuisés par la réforme LMD qu'il a fallu mener à moyens constants, subissent le poids des spécificités nationales et ne mesurent guère la dimension européenne du Processus de Bologne que les classements internationaux tels que celui de Shanghaï ont tendance à minimiser, plaçant ainsi les résultats des activités de recherche au premier plan de la compétitivité. Ainsi les arbitrages entre les fonctions de recherche et d'enseignement sont-ils de plus en plus difficiles à effectuer. C'est sur cette dernière constatation que nous allons centrer notre analyse et montrer que, depuis ces vingt dernières années, les changements multiples qui se sont opérés dans l'enseignement supérieur français ont conduit à générer des tensions de plus en plus fortes à ce niveau. Les reconfigurations du système universitaire proposées sont-elles alors de nature à dépasser les spécificités françaises construites autour de logiques historiques de division?

\section{DES TENSIONS ENTRE LES FONCTIONS ENSEIGNEMENT ET RECHERCHE}

Les lois de 1968 et de 1984 mettent la recherche au centre des missions de l'université. La loi de 1984 définit l'enseignant du supérieur comme un enseignant-chercheur, reconnaissant ainsi une professionnalité duale. Mais cette dualité va de moins en moins de soi, depuis ces vingt dernières années. Plusieurs facteurs peuvent l'expliquer.

\section{Une affaire individuelle}

La structuration de la recherche au sein de l'université reste en effet très faible dans les années quatre-vingts et quatre-vingt-dix : équipes faiblement structurées ou inexistantes, faiblesse des conseils scientifiques, absence de mécanismes d'évaluation. L'adossement de l'enseignement à la recherche est d'abord une responsabilité individuelle de l'enseignant : c'est parce que tout enseignant du supérieur est un enseignant-chercheur que la mise en complémentarité des deux fonctions est considérée comme assurée. La pression collective exercée sur les choix d'orientation de l'activité de recherche de l'enseignant-chercheur est faible; une fois le moment de la thèse passé, cette dernière étant plus abordée dans une démarche de relation interpersonnelle au directeur de thèse et peu en termes d'insertion dans des dynamiques collectives de laboratoires, l'enseignant peut décider d'avoir une activité de recherche ailleurs que dans son université, sans que ce choix soit considéré comme antagoniste au développement de son établissement. Celui qui fait peu ou pas de recherche renonce en partie à un avancement en termes de carrière mais il fait peu l'objet de sollicitations à reprendre un investissement plus actif dans ce domaine. 
Au sein d'un monde universitaire où l'activité de recherche est peu professionnalisée, la façon d'articuler enseignement et recherche est donc surtout un problème de vécu individuel; cette question ne fait l'objet de tensions et de débats collectifs qu'au sein des structures (comme les IUT) qui définissent des objectifs très contraignants en matière de formation, supposant des activités d'encadrement et de suivi des étudiants très consommatrices de temps pour les enseignants-chercheurs et laissant peu de disponibilités pour la recherche.

\section{Massification et professionnalisation}

À partir de 1985, l'enseignement supérieur français a connu une forte croissance de ses effectifs et c'est l'université qui a dû absorber en grande partie le choc de la massification ${ }^{1}$. Cette masse considérable de nouveaux entrants a induit une diversité beaucoup plus grande des étudiants et de leurs attentes. De plus, la montée progressive du chômage a contribué à modifier l'attente des jeunes et de leurs familles à l'égard des formations universitaires. Le diplôme universitaire doit favoriser l'insertion et est perçu comme la meilleure protection contre le chômage. L'importance de la demande de formation universitaire génère une seconde transformation : l'État se révélant incapable de répondre seul aux besoins de financement générés par la pression de la démographie étudiante, il fait le choix d'un financement partagé avec les collectivités territoriales $^{2}$. Ces dernières, qui voient dans l'enseignement supérieur un moyen de revitalisation ou de dynamisation des territoires, favorisent la multiplication des implantations universitaires.

Massification, hétérogénéité des étudiants, angoisse de l'avenir, pulvérisation des implantations universitaires, tel est le nouveau paysage universitaire. Mieux accompagner les étudiants pour limiter l'échec en premier cycle, favoriser les réorientations pour une partie d'entre eux, diversifier l'offre en favorisant la croissance des filières professionnelles deviennent des objectifs des politiques universitaires des années 1995.

Parallèlement à ce mouvement, le ministère mène pendant la même période une politique progressive de structuration de la recherche : incitation à la constitution d'équipes et à leurs regroupements, formalisation plus poussée des écoles doctorales, évaluation externe des politiques scientifiques des établissements et des équipes de recherche. La mise en œuvre de cette politique se fait par le biais des contrats quadriennaux de recherche qui précèdent l'extension de ce mode de conduite de la politique universitaire à l'ensemble de la vie de l'établissement.

1. Formant depuis 1980 près de $70 \%$ à $80 \%$ des étudiants de l'enseignement supérieur, les universités sont ainsi passées de plus de 850000 étudiants en 1980 à 1,5 million en 1995 (incluant IUT et écoles d'ingénieurs universitaires), chiffre relativement stabilisé depuis cette date (Notes d'information de la DEPP, ministère de l'Éducation nationale).

2. Les collectivités territoriales financent généralement le bâti, l’État assumant le financement du fonctionnement. 
Entre une fonction d'enseignement plus difficile à assumer et plus exigeante et une recherche qui se professionnalise, la tension devient plus forte pour un nombre croissant d'enseignants du supérieur. Les activités de formation comme celles de recherche font appel à des dispositifs plus complexes : montage de projets, démarche partenariale, recherche de financements, etc. La prise de conscience de la diversité de situation des enseignants face à l'articulation difficile des composantes du métier génère pour certains une réaffirmation identitaire de l'unicité du métier des enseignants-chercheurs, et pour d'autres une remise en cause de l'unicité de celui-ci avec un appel à la reconnaissance et à la structuration de la gestion de cette diversité.

\section{Shanghaï et au-delà}

Le classement de Shanghaï a été un révélateur de l'intensité de la compétition internationale en matière de recherche et de la difficulté de la recherche française à se situer favorablement au sein de celle-ci. La loi de programme pour la recherche publiée le 18 avril 2006 se veut une réponse à cette situation. L'incitation à la structuration de la recherche est fortement amplifiée, l'évolution de la contractualisation devant permettant de rendre opératoire cette orientation : évaluation et quantification du travail de recherche des équipes dans tous les secteurs (y compris en lettres, sciences humaines et sociales), attribution des moyens sur cette base, lien introduit entre la qualité des équipes de recherche et l'habilitation des formations conduisant aux masters, nécessité beaucoup plus impérative pour les enseignants-chercheurs de développer leur activité de recherche au sein d'équipes (et d'une seule équipe, alors que dans certains secteurs, en particulier en lettres et sciences humaines et sociales, la multiappartenance était encore fréquente), ces éléments ont contribué à accroître la pression sur les universités.

La volonté récente de la direction générale de l'enseignement supérieur du ministère de l'Éducation nationale de «caractériser» la politique de recherche d'une université par des indicateurs quantitatifs et de faire de celle-ci un élément structurant de la négociation contractuelle avec les établissements marque sans doute une nouvelle étape. Les indicateurs retenus ne servent plus simplement à évaluer les équipes mais bien à tracer un profil recherche de chaque université. L'ensemble de cette démarche renforce progressivement le lien entre la qualité du travail de recherche de l'enseignant-chercheur, l'appréciation des équipes et l'appréciation et la caractérisation de l'établissement dans sa politique de recherche globale. La vigilance désormais présente dans de nombreuses équipes de direction universitaire à l'égard des «non-publiants» en est une bonne illustration : détection des «non-publiants», appréciation de leur capacité à reprendre une activité, actions de soutien, incitation à la conversion thématique et éventuellement, pour ceux qui sont désormais trop loin de la recherche, incitation à assurer plus fortement d'autres investissements collectifs; ne pas 
publier n'est plus simplement une caractéristique individuelle pénalisante en termes de carrière mais devient un enjeu collectif d'équipes et d'établissements. L'enjeu "recherche» est plus que jamais au cœur des dynamiques de transformation des universités.

\section{La dynamique européenne}

On peut constater que l'ensemble du discours public incitant les universités à s'adapter s'est articulé sur la nécessité que la France et les universités françaises trouvent leur place dans la dynamique de développement de «l'Europe de la connaissance»; l'idée que les pays européens doivent compenser leurs handicaps par une compétence distinctive dans les domaines de la production, de la diffusion des connaissances et de l'innovation est au cœur du discours politique sur l'enseignement supérieur et la recherche. Si la première traduction de cette orientation a été la mise en place du LMD (licence-masterdoctorat) en France, conçu comme une démarche visant à faciliter la mobilité des étudiants et les partenariats entre établissements par une structuration commune et plus lisible des niveaux de diplômes ${ }^{3}$, il faut cependant constater que les impulsions publiques en France ont principalement concerné l'objectif du renforcement du potentiel compétitif en matière de recherche. D'autres orientations qui relèvent de cette stratégie européenne ont reçu une attention bien moindre; la faible importance accordée, dans les mesures d'impulsion de l'État vers les universités, au développement de la formation tout au long de la vie en est une illustration.

\section{CPE et attentes sociales}

La crise du $\mathrm{CPE}^{4}$ de 2006, comprise comme révélatrice des difficultés d'insertion sur le marché du travail des étudiants ${ }^{5}$, a déplacé récemment l'angle de regard et d'appréciation des universités. Il ne s'agit plus uniquement de compétition et d'excellence en recherche mais aussi de réussite des étudiants, d'orientation, d'acquisition de compétences au-delà de l'acquisition des savoirs, afin de faciliter l'insertion professionnelle.

Les difficultés de fonctionnement du cycle licence sont désormais au centre des interrogations concernant le fonctionnement des universités. Le constat dominant est que le cycle universitaire de licence souffre d'un quadruple déficit : déficit d'orientation, déficit d'attractivité (fortement lié à l'existence des classes préparatoires), déficit de réussite et déficit de qualification et d'insertion

\footnotetext{
3. La structuration du niveau master est sans aucun doute celle qui a le plus retenu l'attention, la réflexion sur le niveau licence se faisant principalement dans l'analyse de sa relation au master.

4. CPE : «contrat première embauche», dérogatoire au contrat à durée indéterminée, visant à faciliter l'obtention d'un premier emploi par les jeunes.

5. Dans les faits, de $7 \%$ à $11 \%$ des diplômés de niveau bac +2 et plus sont au chômage selon les statistiques de l'INSEE et les études du CEREQ portant sur l'enquête «génération 2001 ».
} 
professionnelle, et cela d'autant plus que les entreprises françaises sont souvent plus réticentes que leurs homologues étrangères à recruter des diplômés de filières générales. La nécessité de poursuivre le rapprochement déjà bien engagé entre le monde de l'entreprise et l'université est désormais affirmée, y compris par les organisations étudiantes. De plus, la nécessité de trouver des solutions au problème de l'emploi va probablement accroître l'attention portée à la formation tout au long de la vie; reconnaissance des acquis non formels et développement de la $\mathrm{VAE}^{6}$, formations diplômantes plus ouvertes aux adultes sont et seront des objectifs des politiques publiques et donc des politiques universitaires.

\section{Vers un nouveau paradigme}

L'ensemble de ces attentes suppose que les universités soient attentives à la qualité de ces processus pédagogiques, plus exigeantes sur le suivi personnalisé des étudiants, plus professionnelles dans la définition et la mise en ouvre de partenariats avec le milieu économique, et ce sur l'ensemble de ces niveaux de formation. On retrouve ici les débats européens menés dans le cadre du Processus de Bologne, qui mettent bien en évidence la nécessité de changer de paradigme. Passer d'une démarche centrée sur l'enseignant et les contenus de cours à une approche centrée sur l'étudiant et les résultats apparaît comme un moyen efficace d'adapter et de valoriser les formations universitaires, tant auprès des étudiants que des employeurs. Ainsi, le passage en crédits ECTS ${ }^{7}$ devrait s'accompagner d'une refonte des cursus et méthodes pédagogiques : définir la charge de travail de l'étudiant nécessaire pour atteindre des "résultats de l'apprentissage ${ }^{8}$, attribuer les crédits en fonction des compétences acquises par les futurs diplômés et concevoir de ce fait de nouveaux modes d'évaluation ${ }^{9}$. L'enseignant-chercheur devient alors autant un enseignant qu'un chercheur et l'université un lieu de création et de diffusion du savoir mais aussi de développement des compétences. Toutefois, cette approche nécessite de valoriser à égalité les fonctions enseignement et recherche, ce qui est loin d'être le cas actuellement dans les universités françaises.

\section{Le poids de la dualité université / grande école}

Les exigences accrues de qualité à l'égard de l'université, en ce qui concerne la formation, ne font pas disparaître les adaptations nécessaires en recherche pour faire face à un environnement plus concurrentiel. Si la mise en

6. Validation des acquis de l'expérience.

7. European Credit Transfer System.

8. La traduction de ce terme et de ce concept anglo-saxon n'étant guère consensuelle, de nombreux pays européens ont opté pour le maintien de l'expression anglaise Learning outcomes (LO).

9. Ainsi l'expression même de «contrôle des connaissances» devra-t-elle être repensée. 
cohérence entre ces deux transformations est plus facile pour le niveau master, elle est plus complexe pour le niveau licence par la juxtaposition d'une double caractéristique : diversité des étudiants et enseignement de masse. Cette dernière génère des tensions à la fois au niveau individuel (quelle différenciation dans les modes d'exercice du métier d'enseignant-chercheur?), mais aussi au niveau de la stratégie globale de l'établissement. Si cette situation n'est pas propre aux universités françaises, la structuration du système d'enseignement supérieur français, qui présente une double particularité, ne facilite pas les adaptations :

- coexistence en premier cycle d'une structure ouverte, le cycle licence des universités, et de structures sélectives, soit internes aux universités (les IUT), soit externes comme les classes préparatoires aux grandes écoles ou les sections de technicien supérieur;

- juxtaposition des universités et des grandes écoles, l'essentiel de la fonction de formation des élites étant assuré par ces dernières, la recherche étant significativement plus présente dans les universités qu'au sein des grandes écoles.

La recherche de l'équilibre entre les exigences de compétition en recherche et l'amélioration de la qualité en formation, en particulier au niveau de la licence, est d'autant plus difficile que les universités accueillent en premier cycle une part importante des étudiants les plus fragiles, les moins adaptés à une formation universitaire "classique». L'université reste en effet en France, contrairement à la majorité des pays ${ }^{10}$, la deuxième voie d'accès aux études supérieures ${ }^{11}$. Les meilleurs bacheliers choisissent les filières sélectives des classes préparatoires et les meilleurs diplômés de licence ou de première année de master des filières scientifiques et de gestion universitaires ont tendance à vouloir intégrer une grande école, les niveaux d'insertion professionnelle puis de carrière étant encore nettement plus avantageux.

\section{VERS UNE RECONFIGURATION DU PAYSAGE UNIVERSITAIRE?}

Les adaptations attendues des universités doivent être regardées en prenant en compte les transformations opérées dans les décennies quatre-vingts et quatre-vingt-dix évoquées précédemment.

\section{Des stratégies de différenciation des formations}

En formation, les universités ont très souvent répondu à la pression de la démographie étudiante et aux attentes des collectivités territoriales, acteurs devenus essentiels du développement universitaire, par une stratégie systématique

10. Par exemple, plus de $60 \%$ des diplômés de l'enseignement supérieur aux États-Unis sont formés dans des Community colleges (formation en deux ans), les deux tiers dans des Hogeschool aux Pays-Bas.

11. Sauf pour les formations juridiques, médicales, de pharmacie et d'odontologie. 
de différenciation des formations, en particulier des formations professionnalisées. La différenciation des formations a souvent été très poussée, les universités multipliant les formations dans des domaines proches, en particulier (mais pas exclusivement) dans le secteur tertiaire. Si cette stratégie a été largement plébiscitée par les étudiants, en particulier au niveau des anciens DESS devenus masters professionnels, elle a certainement contribué à rendre plus délicate l'articulation recherche/formation. Les risques d'une différenciation excessive de l'offre de formation sont probablement les suivants :

- une taille restreinte des équipes d'animation des formations;

- une plus grande difficulté d'articulation des thématiques de recherche avec cette forte diversité des champs couverts en formation.

La nécessité affirmée par de nombreux acteurs publics d'atteindre une taille critique, base de la capacité compétitive en recherche, est perçue par de nombreuses universités comme une remise en cause du modèle précédemment développé par elles (au niveau des directions et des enseignants-chercheurs), les plus jeunes étant souvent les plus innovatrices pour améliorer la qualité de la formation.

\section{Vers une stratification des universités?}

La première réponse possible aux conditions de l'environnement universitaire est une stratification des universités, sous l'effet de la concurrence, entre universités à dominante recherche et universités à dominante formation. Comme Jean-Richard Cyterman le note, on peut considérer que de nombreux indices montrent déjà l'existence de cette différenciation entre universités : «la recherche universitaire est ainsi déjà extrêmement concentrée : quinze universités reçoivent plus de $50 \%$ des moyens (salaires des enseignants-chercheurs et apport des grands organismes inclus). Dans chaque grand secteur disciplinaire, une dizaine d'universités fournit $60 \%$ des docteurs recrutés comme maîtres de conférences. Certaines des universités ont plus de $80 \%$ de leurs effectifs étudiants et assurent prioritairement un enseignement de proximité; d'autres ont construit leur identité sur des filières professionnelles comme Valenciennes et Mulhouse. D'autres sont plutôt des universités à dominante recherche, dans les grandes métropoles universitaires ${ }^{12}$

Si cette évolution peut apparaître comme étant la plus naturelle, voire pour certains souhaitable et inéluctable, il faut constater que le modèle recherché par les autorités politiques et les responsables d'universités n'est pas celui-ci. Trois raisons probablement à ce refus :

- cette stratification remet en cause radicalement la vision que les enseignants-chercheurs se font de leur professionnalité, l'accès à la fonction de

12. Communication tenue lors du colloque «Internationalisation de l'enseignement supérieur» organisé par I'Université du Littoral en novembre 2006. 
recherche étant perçu comme l'élément essentiel de leur reconnaissance et de leur appartenance; cette évolution peut alors être considérée comme socialement (et donc politiquement) difficile;

- la stratification introduit une césure entre universités des grandes métropoles des centres et universités des villes moyennes et de périphérie parisienne; elle est donc aussi une césure territoriale, difficilement acceptable pour les collectivités territoriales qui ont soutenu financièrement leurs universités;

- mais, probablement aussi parce que toute université présente en son sein des secteurs d'excellence en recherche, plus ou moins nombreux, et des secteurs structurés principalement par la formation et où la relation à la recherche est d'une autre nature que celle observée dans les premiers secteurs, favoriser (ou laisser apparaître par le jeu concurrentiel) une stratification des universités à dominante recherche et d'autres à dominante formation supposerait une recomposition profonde du tissu universitaire et des frontières institutionnelles des établissements; on peut supposer que cela entraînerait à la fois du temps et de fortes tensions pour la mise en ouvre.

Enfin, soulignons que ce qui différencie précisément l'université d'un autre établissement d'enseignement supérieur dans le monde entier est bien la capacité à articuler la formation et la recherche. Les débats en cours à l'EUA ${ }^{13}$ autour des règles à fixer pour adhérer à cette association sont à cet égard très importants à suivre ${ }^{14}$.

\section{Des stratégies de coopération entre établissements?}

La stratégie aujourd'hui privilégiée pour la transformation du paysage universitaire français est basée sur le développement de la coopération entre établissements. La mise en place de structures fédératives inter universités et/ou inter écoles que constituent les PRES (pôles régionaux d'enseignement supérieur) est conçue comme devant permettre :

- le regroupement des équipes de recherche afin d'atteindre la taille critique ;

- une visibilité d'ensemble plus repérable et s'appuyant sur la mise en valeur, au niveau international, de formations doctorales et de masters communs d'excellence;

- le développement de fonctions supports partagées entre établissements associés dans le PRES et devant permettre d'améliorer la qualité et l'efficacité de la recherche et de la formation : développement international, ingénierie

13. EUA : association européenne de l'université (European University Association).

14. Les discussions portent sur la nécessité de répondre au moins à trois critères parmi lesquels : la délivrance de formations doctorales, la participation active à des écoles doctorales avec des établissements habilités, au moins $50 \%$ du corps enseignant titulaire d'un doctorat, le développement de programmes de recherche ou la participation active à des programmes de recherche (www.eua.be). 
de projet, valorisation de la recherche, suivi et appui de l'insertion des étudiants... Il faut noter que ces fonctions sont souvent à l'articulation des universités et de leurs environnements et supposent une capacité à imaginer et mettre en œuvre des partenariats.

Cette stratégie coopérative ne fait pas disparaître comme par miracle les problèmes d'articulation entre enseignement, fonction d'accueil en premier cycle, fonction de proximité d'une part et fonction de recherche d'autre part. Elle peut permettre de rechercher les adaptations plus progressivement, de favoriser des recouvrements entre les deux dynamiques plus facilement que dans l'hypothèse d'une coupure institutionnelle. Elle peut aussi favoriser une gestion dans le temps des investissements enseignants avec des moments plus nettement dédiés à la recherche, d'autres plus tournés vers l'enseignement ou l'animation des équipes. Elle suppose de promouvoir également excellence en recherche et qualité en formation, avec un même niveau d'exigence et de reconnaissance.

La taille des regroupements ainsi obtenus (fréquemment trente à quarante mille étudiants) demande cependant d'être attentif à la gestion opérationnelle des structures, en distinguant bien ce qui doit relever de la gestion de chaque université pour des raisons de souplesse et ce qui doit appeler une action commune; au fond, comment combiner un niveau de mise en cohérence stratégique et un niveau plus opérationnel. Enfin, si le PRES est le lieu de la définition et de la mise en œuvre des stratégies communes, cela supposera cependant que les directions d'universités trouvent les moyens de faire partager les enjeux au sein de chaque université. Dans le cas contraire, les risques de tensions entre les deux niveaux seront probablement très forts. La relation PRES/universités est d'une autre nature que celle existant entre maisons mères et filiales dans le monde économique. Cette nouvelle dimension ajoutera certainement à la complexité du management universitaire.

La stratégie coopérative est aussi considérée comme un moyen de dépasser la coupure université/grande école. Plusieurs tentatives de réforme, depuis la fin du XIX ${ }^{e}$ siècle, relancent régulièrement le débat. En 1998, le rapport Attali soulignait que «lorsque les universités auront rattrapé leur retard financier, on pourra intégrer les classes préparatoires en leur sein ». Dix ans plus tard, aucun effort public n'a été fait pour rattraper ce retard et force est de constater que malgré leur volonté de "s'universitariser» en recrutant davantage de docteurs et en investissant dans la recherche, les grandes écoles ne sont pas encore assez visibles à l'échelle internationale. Or nous savons que l'interdépendance des deux systèmes est forte. Comment pourrait-on alors imaginer de réussir une nouvelle réforme des universités sans envisager des changements majeurs pour l'ensemble du système d'enseignement supérieur? La question est-elle alors d'intégrer les classes préparatoires et les écoles aux universités, de leur conférer le statut d'université dès lors qu'elles remplissent les missions définies par la loi, ou/et de favoriser des partenariats à travers les PRES nouvellement 
mis en place? La réponse est loin d'être aisée mais doit être clairement posée. Un responsable de spécialité de master recherche de la première université française apparaissant au classement de Shanghaï nous parlait de son inquiétude lorsqu'il voyait partir ses meilleurs étudiants de licence et de première année de master dans les écoles, lorsque le recrutement de thésards est de plus en plus difficile alors qu'il y a peu de temps encore, les meilleurs diplômés des grandes écoles venaient s'inscrire dans ce master recherche pour faire une thèse par la suite. Certes les laboratoires sont fréquemment co-habilités mais la possibilité de former de futurs bons chercheurs à l'université dès le premier cycle semble se réduire de plus en plus. Or les meilleures universités du monde (en tout cas les plus recherchées) ne le sont-elles pas aussi précisément grâce à leur premier cycle (Rosovsky, 1991)?

Faut-il alors ralentir ou favoriser une plus forte hiérarchisation du système pour sauver la mission mondialement reconnue de l'université, à savoir l'articulation équilibrée entre la formation et la recherche? Ce débat, plus fort que jamais en Europe, est désormais public en France.

\section{BIBLIOGRAPHIE}

ATTALI Jacques (1998): Pour un modèle européen d'enseignement supérieur, Stock. GIRET Jean-Pierre, MOLINARI-PERRIER Mickaëlle, MOULLET Stéphanie : «20012004, les sortants de l'enseignement supérieur face au marché du travail», Notes Emploi Formation (NEF), n 21, mars 2006, Céreq.

JOURDE Pierre et al. (2007): Université : la grande illusion, L'esprit des péninsules. MILOT Pierre: «La redéfinition du rôle de l'enseignement supérieur dans l'économie du savoir des pays de l'OCDE», Bulletin de l'enseignement supérieur (Groupe de recherche sur l'enseignement supérieur), vol. 6, n 1 , octobre 2001.

ROSOVSKY Harry (1991): The university, an owner's manual, Norton Paperback. SCHIMANK Uwe (2007): "New public management and the academic profession - Reflections on the German situation». Minerva, vol. 43, $\mathrm{n}^{\circ}$ 4, décembre 2005, pp. 361-376. 
